1 Article

\title{
The Effect of Temporal Gradient of Vegetation Indices on Early-Season Wheat Area Estimation Using Random Forest Classification
}

\author{
Mousa Saei Jamal Abad ${ }^{1}$, Ali A. Abkar ${ }^{2}$ and Barat Mojaradi ${ }^{3, *}$ \\ ${ }^{1}$ Faculty of Geodesy and Geomatics, K. N. Toosi University of Technology, Tehran 19667-15433, Iran; \\ msaei@mail.kntu.ac.ir \\ ${ }^{2}$ Faculty of Geodesy and Geomatics, K. N. Toosi University of Technology, Tehran 19667-15433, Iran and \\ AgriWatch BV, Enscede, the Netherlands ; ali.abkar@agriwatch.nl \\ 3 Department of Geomatics, School of Civil Engineering, Iran University of Science and Technology, Tehran, \\ Iran ; mojaradi@iust.ac.ir \\ * Correspondence: mojaradi@iust.ac.ir; Tel.: +98-912-215-2274
}

\begin{abstract}
The early-season area estimation of the winter wheat crop as a strategic product is important for decision makers. Classification of multi-temporal images is an approach which is affected by many factors like appropriate training sample size, proper frequency and acquisition times, vegetation indices (VIs) type, temporal gradient of spectral bands and VIs, appropriate classifier and missed values because of cloudy conditions. This paper addresses the impact of appropriate frequency and acquisition times and VIs type along with the spectral and VI gradient on random forest $(\mathrm{RF})$ classifier when missed values exist in multi-temporal images. To investigate the appropriate temporal resolution for image acquisition, the study area was selected on an overlapping area between two LDCM paths. In our developed method, the miss values of cloudy bands for each pixel are retrieved by the mean of k-nearest ordinary pixels. Then the multi-temporal image analysis is performed by considering different scenarios provided by decision makers in terms of desired crop types that should be extracted at early-season in the study areas. The classification results obtained by the RF decrease by $1.6 \%$ when temporally missed values retrieved by the proposed method, which is an acceptable result. Moreover, the experimental results demonstrated that if temporal resolution of Landsat 8 increased to one week the classification task can be conducted earlier with almost better results in terms of OA and kappa. The impact of incorporating VIs along with the temporal gradients of spectral bands and VIs as new features in RF demonstrated that the OA and Kappa are improved 3.1\% and 6.6\%, respectively. Furthermore, the obtained result showed that if only one image from seasonal changes of crops is available, the temporal gradient of VIs and spectral bands play the main role to discriminate remarkably wheat from barley. The experiments also demonstrated that if both wheat and barley merge to a single class the crop area can be estimated two months earlier with 97.1 and 93.5 in terms of OA and kappa, respectively.
\end{abstract}

Keywords: Wheat classification; Random Forest; Spectral gradient difference; Vegetation indices

\section{Introduction}

The global demand for food will increase due to increasing of the global population in this century [1]. It is therefore necessary to accurately estimate the crop area to determine if the demand for food can be met [2]. Farmers usually decide to change the crop type of their agricultural fields due to the regional demand and drought condition. Therefore, the cultivated area of a given crop alters from a growing season to next season. Consequently, early-season crop area forecasting, in particular wheat crop, is an essential information for policy makers to manage yields for domestic use, imports or exports. 
The classification of remotely sensed data can provide regional distribution of individual crop types for government agencies [3,4] and decision makers of commodity trade. Evidently, the classification of agricultural crops using only one image captured at a given date has significant shortcomings [5]. Firstly, the crop types may have different growth stages and secondly, different crops may have similar spectra (e.g. wheat and barley in this case study) during the growing season in a complex agricultural area [6]. It is therefore difficult to distinguish crop types at a certain point in the growth cycle by an image in a given time. In contrast, multi-temporal images provide temporal signatures of crops and represent the growth cycle by capturing data during the growing season [7]. Therefore, the temporal signatures of crops may be considered to discriminate crop types [8], and maps of cropland distributions are usually obtained by the supervised classification of multitemporal images throughout the growing season $[9,10]$. A significant advantage of using multitemporal data is that the proportions of different crop types can be discriminated using the spectral differences at different points in the growing season [6,11,12]. Recently, crop areas from non-crop areas have been separated by detecting changes in greenness using time-series normalized difference vegetation index (NDVI) data in [13]. Moreover, an approach based on object-based Normalized Difference Vegetation Index (NDVI) time series analysis is used for regional-scale mapping of agricultural land-use systems [14]. The crop area in early-season was also determined using enhanced vegetation index (EVI) of multi-temporal Moderate Resolution Imaging Spectroradiometer (MODIS) in $[11,15]$. In their study, the green-up rate of the crop canopy, i.e. the accumulated difference of two consecutive images, is used. The obtained results indicated that multi-temporal remote sensing approach could be used with confidence in early-season crop area prediction at least one to two months ahead of anthesis. Moreover, with increasing number of images, applying different VIs and their differences, the training sample size increases dramatically to overcome the curse of dimensionality for supervised classification [16]. In the study which conducted by [17] different data types including reflectance data, spectral indices, texture indices and DEM features are used in a combined random forest (RF) and object bases image analysis scheme for agricultural area mapping. But these approaches require cloud-free imagery and processing of multiple images is prohibitive for operational implementation over large areas. In this regard, acquiring such frequent cloud-free imagery is a serious limitation in estimating crop area $[18,19]$

Indeed, classification of multi-temporal images is a challenging task which is affected by many factors that limit the accuracy. In particular, these issues are appropriate training sample size, proper frequency and acquisition times and suitable VIs. The effect of these factors also depends on the choice of the classifier [20]. Some studies are conducted to address some of these issues[21-24]. For example, the optimal number and dates of images for land cover classification are identified with feature importance yielded by RF classifier on remote sensing time-series [25]. In another study the sensitivity of the RF classification to select training sample points, including sample size, spatial autocorrelation and proportions of classes within the training sample were investigated [21]. In [26] the effect of the time series length on crop mapping is investigated. In this study RF is used to calculate the important scores for VIs and Jeffries -Matusita (JM) distance is used to measure class separability for each time series. In a comprehensive study also five strategies that can be applied to high resolution multi-temporal optical imagery to produce accurate crop type maps have been selected and benchmarked at the global scale [27]. These strategies are RF, support vector machine (SVM) with a redial basis function kernel, Dempster-Shafer fusion of the previous approaches, best classifier with a mean-shift smoothing, best classifier with a temporal regular resampling. The obtained classification results on the 12 test sites over the globe demonstrated that RF achieved superior results. The RF also is used for winter wheat mapping by multi temporal images of LDCM and Gao Fen 1 satellites [28].

In aforementioned circumstances, the study of optimal number of images to find critical acquisition times and temporal resolution along with VIs and their gradient is necessary. This paper will address to this issue that how much importance the temporal resolution of image acquisition for multi temporal images classification and what happen when temporal resolution of Landsat 8 increase to one week? For this purpose, the study area is selected on overlap area between two 
Landsat paths. Moreover, missed values problem in cloudy times is another matter which limits the application of multi-temporal image classification. Indeed, many pixels of training or non-training data are ignored if only one cloudy image exist in the time series. Although considerable research has been previously conducted on the time-series classification, the problem of miss values caused by cloudy pixels in such images is still an issue to which less attention has been paid. Indeed, the pixels of data are either cloudless pixels or miss data pixels associated with miss values (i.e. bands with no data) due to the availability of cloudy images in time series. One of the main problems in the multitemporal image classification is that some pixels of training/testing areas may be covered by cloud during the analysis period. Hence, those pixels are ignored and neither incorporated as training data in the classifier learning process nor as test data for the classification accuracy. On the other hand, the rest of cloudy pixels cannot be classified by classifier. This paper remedies this problem for those training/test data and for rest of cloudy pixels by retrieve of the miss values trough k-nearest neighbor cloudless pixels. This paper proposes a method to retrieve the miss values and study the effect of miss value retrieval of training data on classification results of multi temporal images.

This paper also addresses the impact of the appropriate frequency and acquisition times and VIs along with the spectral and VI gradient on the RF classifier when miss values are available in the multi-temporal images. The objective of this paper is therefore to investigate the practical considerations of the multi-temporal image classification approach for consultants and agronomists. The specific goals of this study are: (1) To assess the effect of miss values in training areas, due to cloudy times, on RF classification learning; (2) To determine the appropriate data acquisition times and temporal resolution; (3) To assess the effect of VIs and their temporal gradient along with temporal gradient of spectral bands in different times; and (4) To determine the optimum number of classes to be distinguished in early season. To our knowledge, there are currently no other comprehensive assessments of multi-temporal image classification which takes into consideration these practical aspects.

\section{Materials and Methods}

2.1. Study Area and Data: The study area is an agricultural region with an area of $755 \mathrm{~km} 2$, located between latitudes $29^{\circ} 49^{\prime}-30^{\circ} 05^{\prime} \mathrm{N}$ and longitudes $52^{\circ} 39^{\prime}-52^{\circ} 55^{\prime} \mathrm{E}$, in Southwest of Iran (Figure 1. The area has various climates during the growing season and is covered by different vegetation, soil, and rocky terrain types. The study area is situated on overlapping area between two paths of 162 and 163, which is covered by 26 scenes of Landsat Data Continuity Mission (LDCM) data captured from 8 Nov 2014 until 26 May 2015 during growing season. In this regard, the temporal resolution in the study area increases from 2 weeks to one week to investigate the appropriate temporal resolution for image acquisition. Among all images, 10 images having less than $20 \%$ cloud in the study area are selected which 8 images belonging to path 162 and 2 images to path 163 . The acquisition times of these images are given in Table 1.

Table 1. Acquisition times of images.

\begin{tabular}{cccc}
\hline Image No. & Acquisition Date & Image No. & Acquisition Date \\
\hline T1 & 11-JAN-15 & T6 & 17-APR-15 \\
\hline T2 & 03-FEB-15 & T7 & 03-MAY-15 \\
\hline T3 & 28-FEB-15 & T8 & 19-MAY-15 \\
\hline T4 & 07-MAR-15 & T9 & 04-JUN-15 \\
\hline T5 & 16-MAR-15 & T10 & 20-JUN-15 \\
\hline
\end{tabular}




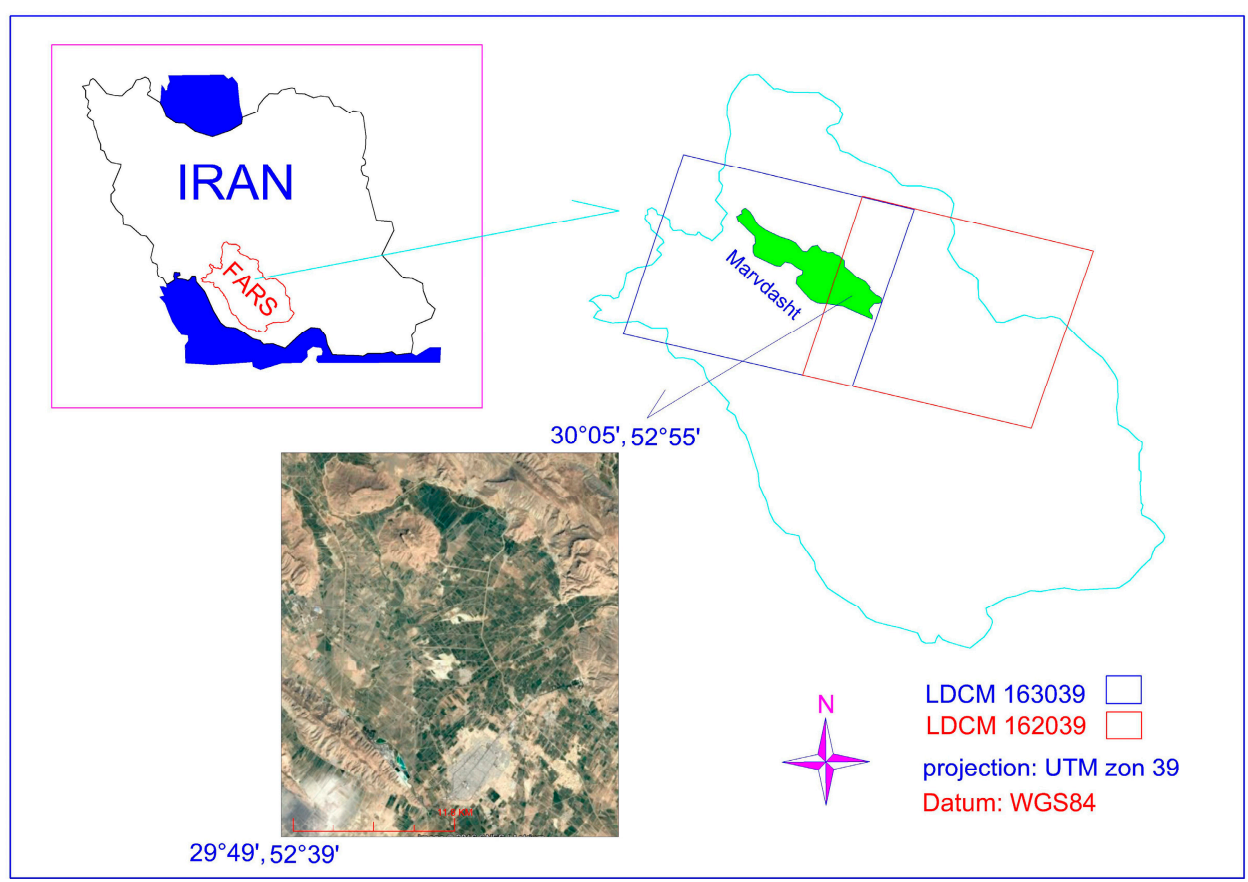

Figure 1. The study area situated in the overlap area between two paths 162 and 163 of LDCM.

138 139 140 141

The training data also were captured in 264 agriculture fields. The spatial distribution of the training and test areas for the three classes of wheat, barley and other-classes are given in Figure 2 and Table 2. As shown, training and test data are selected in disjoint agricultural fields to consider real situation.

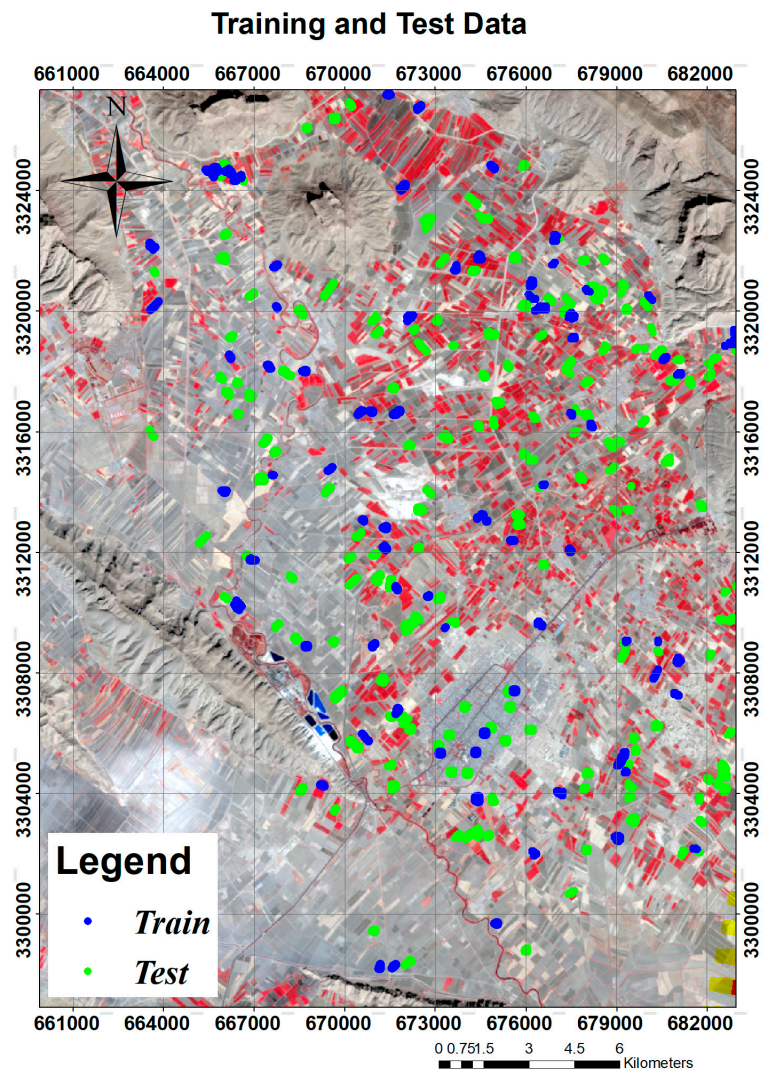


Table 2. The number of training and test data in the study area.

\begin{tabular}{ccccc}
\hline Class & \# of training fields & \# of test fields & \# of training pixels & \# of test pixels \\
\hline Wheat & 128 & 280 & 559 & 1265 \\
\hline Barely & 15 & 51 & 93 & 307 \\
\hline Other & 121 & 252 & 1419 & 3027 \\
\hline
\end{tabular}

\section{5}

146

147

148

149

150

151

152

153

\subsection{Methodology}

As mentioned above, the classification of multi-temporal images is affected by many factors such as miss values in cloudy images, optimum training sample size, proper frequency and acquisition times that limit the accuracy. Moreover, extraction of appropriate features (e.g. temporal gradient) from the temporal signatures of crop types along with suitable times such that discriminate crops are two major issues that should be considered in the multi-temporal image classification. In this regard, different features like VIs and the temporal gradient of VIs and spectral bands are taken into account as new features. To address aforementioned issues, we developed following methodology (Figure 3).

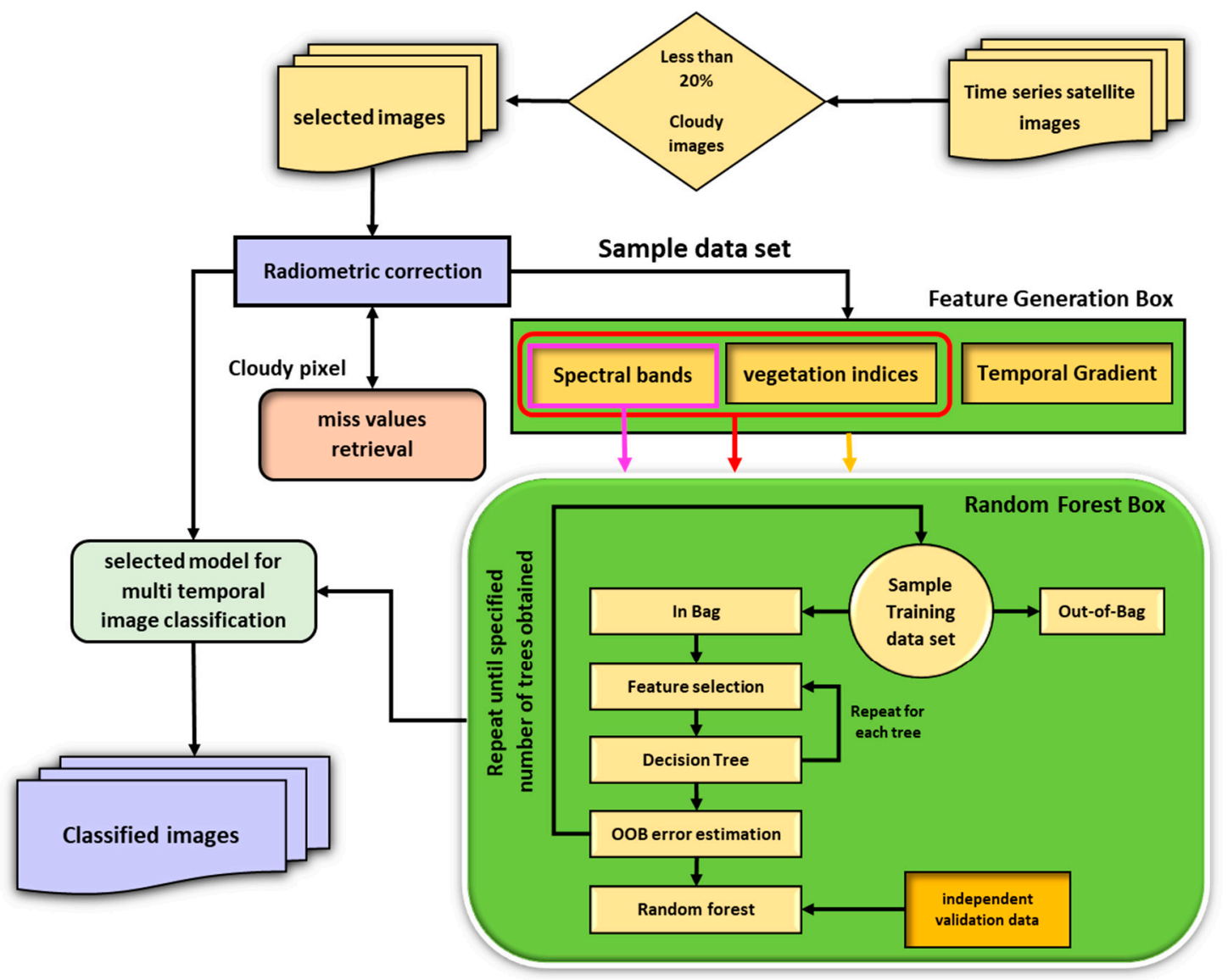

Figure 3. Flow chart describing the method developed in this study for multi-temporal image classification

Step 1: A set of images with acceptable cloud percent, $20 \%$ in this study, is selected from input time-series images. Radiometric correction is then conducted on the selected images (i.e. 10 images for this study area) and cloudy pixels in each image are set to no data as missed values for all bands. Hence, for each pixel, a feature vector with a dimension of 80 elements $(8 \times 10)$ is achieved.

Step 2: The missed values problem limits the use of training/test data to learn the classifier and the rest of cloudy pixels also cannot be classified in the imaging scene. To overcome this shortcoming for ground data, the bands of a pixel corresponding to cloudy times in a given training/testing class 
163 (e.g. wheat), which contain no data are removed from the feature vector and flagged as cloudy pixels. 164 The corresponding bands from the feature vector of the cloudless pixels of that class are also removed 165 (i.e. the corresponding bands of cloudy times are removed in cloudless pixels of that class) to achieve 166 the same dimensionality with the cloudy pixels. In the reduced multi-dimensional feature space, for 167 each cloudy pixel, k-nearest neighbor cloudless pixels are then found. Therefore, the bands of those 168 pixels with no data are reconstructed by mean of k-nearest cloudless pixels to yield a feature vector 169 with full dimensionality. In other words, the miss values of cloudy bands are filled with the mean of 170 k-nearest from cloudless pixels which are spectrally similar to cloudy pixels in feature space. This is 171 also conducted for the whole cloudy pixels of the scene after the appropriate multi-temporal 172 classification model is selected.

Step 3: After miss value retrieval, the VIs are generated. In the current study 9 indices including NDVI, SAVI, SR, EVI, STVI1, STVI3, STVI4, MSAVI1 and MNDVI are generated based on the corresponding equation listed in Table 3 , as new features.

Table 3. Remote sensing vegetation indices used in this study.

\begin{tabular}{|c|c|c|c|}
\hline No. & Index & Formula & Reference \\
\hline 1 & $\begin{array}{c}\text { Normalized Difference } \\
\text { Vegetation Index }\end{array}$ & $N D V I=N I R-R / N I R+R$ & [29] \\
\hline 2 & Simple Ratio & $S R=N I R / R$ & [30] \\
\hline 3 & $\begin{array}{c}\text { STress related } \\
\text { Vegetation Index } 1\end{array}$ & $S T V I 1=M I R \times(R / N I R)$ & [31] \\
\hline 4 & $\begin{array}{c}\text { STress related } \\
\text { Vegetation Index } 3\end{array}$ & $S T V I 3=N I R /(R+M I R)$ & [32] \\
\hline 5 & $\begin{array}{c}\text { STress related } \\
\text { Vegetation Index } 4\end{array}$ & $S T V I 4=N I R-\frac{R \times M I R}{N I R+M I R}$ & {$[33,34]$} \\
\hline 6 & $\begin{array}{l}\text { Enhanced Vegetation } \\
\text { Index }\end{array}$ & $E V I=2.5 \times\left(\frac{N I R-R}{N I R+6 R-7.5 B+1}\right)$ & {$[8,35]$} \\
\hline 7 & $\begin{array}{l}\text { Modified Soil Adjusted } \\
\text { Vegetation Index }\end{array}$ & $M S A V I=\frac{(2 N I R+1 ? \sqrt{(N I R+1) 2 ?(N I R ? R)})}{2}$ & [36] \\
\hline 8 & $\begin{array}{c}\text { Modified Normalized } \\
\text { Difference Vegetation } \\
\text { Index } \\
\end{array}$ & $M N D V I=N D V I \times \frac{\left(S W I R_{\max }-S W I R\right)}{\left(S W I R_{\max }+S W I R_{\min }\right)}$ & [37] \\
\hline 9 & $\begin{array}{c}\text { Soil Adjusted } \\
\text { Vegetation Index }\end{array}$ & $S A V I=\frac{N I R-R}{N I R+R+L}(1+L)$ & [38] \\
\hline
\end{tabular}

Step 4: Because temporal signature plays an important role in the interpretation of the growing steps of crops, temporal gradient is used to quantitatively describe the seasonal change. The temporal gradient of a given spectral band, TGSB, at the ith $\left(t_{i}\right)$ and $j$ th $\left(t_{j}\right)$ times are then generated to obtain the temporal changes of crops as follows.

$$
\operatorname{TGSB}\left(t_{i}, t_{j}\right)=\mathrm{SB}\left(t_{j}\right)-\mathrm{SB}\left(t_{i}\right)
$$

The temporal gradients of VIs, TGVI, are also computed and considered as new features, which means for a given VI at the ith $\left(t_{i}\right)$ and $\mathrm{jth}\left(t_{j}\right)$ times, a set of VI gradients in time sequence is generated as follows:

$$
\operatorname{TGVI}\left(t_{i}, t_{j}\right)=\mathrm{VI}\left(t_{j}\right) \text { - VI }\left(t_{i}\right)
$$

Step 5: The RF algorithm is conducted to explore the achieved features including spectral bands at different times, VIs and their temporal gradients to yield an ensemble of decision trees. As mentioned, 8 images from path 162 and 2 images from path 163, having less than 20\% cloud cover 
190

191

192

193

194

195

196

197

198

199

200

201

202

203

are selected. Therefore, for each pixel, a feature vector is obtained that includes spectral bands, VIs along with their temporal gradients. To investigate the effect of multi-temporal image classification issues by the RF classification, different scenarios are considered according to Table 4 .

The RF algorithm can generally handle high dimensional data and use a large number of trees in the ensemble. In RF, each tree in the forest is built and tested independently from other trees. During training, each tree receives a new bootstrapped training set generated from the original training set by subsampling with replacement [39]. Those samples which are not included during the training of a tree are called Out-Of-Bag (OOB) samples of that tree. These samples can be used to compute the Out-Of-Bag-Error (OOBE) of the tree as well as the ensemble which is an unbiased estimate of the generalization error [40-42].

In the experiments, replacement and OOB are set to 15 times and $33 \%$, respectively. First, the optimum number of training sample size along with the number of trees are investigated. Then, the effect of each issue is assessed to achieve an appropriate model for the multi-temporal classification.

Table 4. The different scenarios for the RF classification.

\begin{tabular}{ccc}
\hline \multicolumn{3}{c}{ Experimental setup } \\
\hline Forest size (\# of trees) & \multicolumn{2}{c}{10 up to 80} \\
\hline Training sample size & $10 \%$ up to $50 \%$ \\
\hline Miss values & Yes & No \\
\hline VIs & Yes & No \\
\hline Temporal gradient of VIs and spectral bands & Yes & No \\
\hline \# Images & \multicolumn{2}{c}{2 up to 10} \\
\hline
\end{tabular}

\section{Results and Discussion}

Indeed, decision makers aim at achieving crop area from remotely sensed data in earlier season with appropriate accuracy. Therefore, according to Table 5, based on the desired classes two cases of classification are determined by the decision makers.

Table 5. Two cases of classification.

\begin{tabular}{ccc}
\hline Case & \# of classes & desired classes \\
\hline 1 & 3 & wheat, barely, other \\
\hline 2 & 2 & (wheat \& barely), other \\
\hline
\end{tabular}

\subsection{Experiment 1: Optimum Training Sample Size}

In this experiment the sample size of the training data, set to $10 \%$ up to $50 \%$ without considering

211 missed values and only spectral bands of 8 images captured on path 162, without their gradient and

212 VIs are used for classification. The RF classification is performed with 15 times replacement and with

21350 trees (forest size) in case 1. The averages of the overall accuracy (OA) and Kappa coefficient [43]

214 are computed and given in Figure 4. 


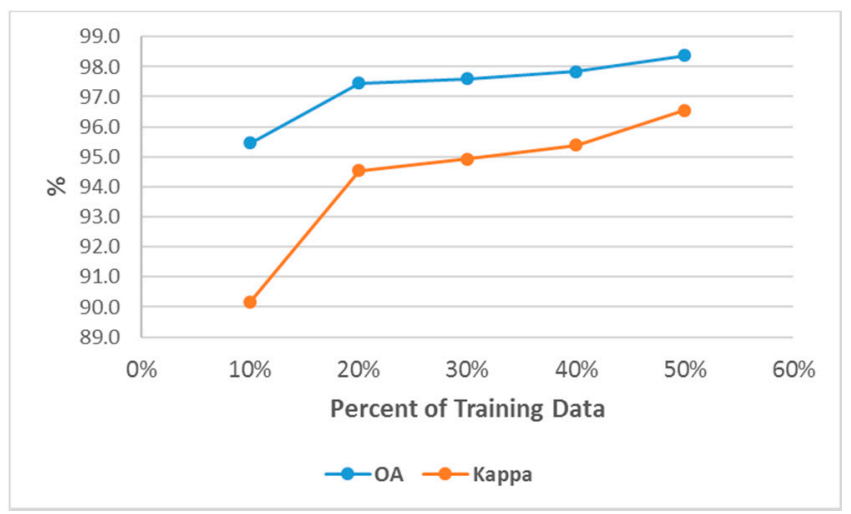

Figure 4. Overall accuracy and Kappa coefficient changes to obtain optimum training sample size.

With increasing training sample size, the OA and the Kappa coefficient are improved (Figure 4). This improvement is not remarkable when the training sample size become more than $30 \%$. Hence, we selected $30 \%$ as appropriate training size due to the cost consuming of filed works.

\subsection{Experiment 2: Optimum Forest Size}

Most of experiments used RF classification are conducted with the large number of trees which is time consuming[26,44]. Hence, the optimum forest size for each dataset should be investigated and determined. For this purpose, after achieving the optimum sample size in experiment 1 , the optimum forest size is also investigated through increasing trees from 10 up to 80 trees. In this experiment, the RF classification is also conducted with 15 times replacement in case 1 using only the spectral bands of all images. The average values of OAs are given in Figure 5. As demonstrated in Figure 4, the OA becomes almost steady when the number of trees exceeds 50 . Hence in this dataset, the optimum forest size and optimum number of training sample size set to 50 trees and $30 \%$, respectively.

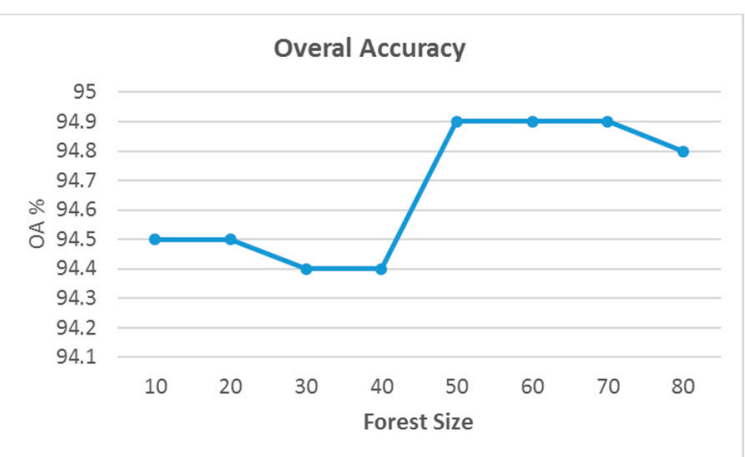

(a)

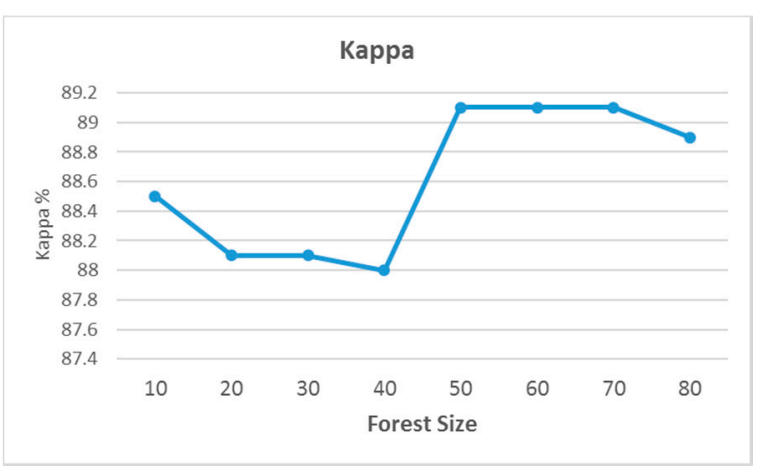

(b)

Figure 5. Effect of the number of trees on (a) overall accuracy (OA) and (b) Kappa coeficient

\subsection{Experiment 3: The Effect of Miss Values in the Training Data}

In fact, the pixels of ground data in some images are cloudless while at other times they may be cloudy. In multi-temporal image classification, keeping these pixels is valuable in terms of being time and cost consuming. Moreover, the classification of those pixels which have missed values due to the cloudy times is important when multi-temporal images are used. As mentioned is step2 of the methodology, the miss values (i.e. cloudy bands) of pixels are replaced by the average of k-nearest cloudless pixels. To investigate the effect of the data retrieval method, the retrieval cloudy pixels of the ground data are incorporated to train the RF. Moreover, the retrieval cloudy pixels also are incorporated in test data and are classified. Table 6 shows that the OA value decreases by $1.6 \%$ due to the retrieval miss values by the mean of k-nearest neighbor algorithm. Therefore, it can be 
the certainty of obtaining labels for these pixels are less than those of cloudless pixels. Note that all time-series images are used in this experiment and $\mathrm{k}$ is set to 7 in this study.

Table 6. The effect of miss values on the overall accuracy in the RF classifier.

\begin{tabular}{cccccc}
\hline miss value & OA & Kappa & $\begin{array}{c}\text { Product Accuracy } \\
\text { wheat }\end{array}$ & $\begin{array}{c}\text { Product Accuracy } \\
\text { barely }\end{array}$ & $\begin{array}{c}\text { Product Accuracy } \\
\text { others }\end{array}$ \\
\hline Yes & 93.2 & 85.3 & 91.5 & 51.1 & 97.7 \\
\hline No & 95.4 & 90.3 & 96.5 & 62.6 & 98.0 \\
\hline
\end{tabular}

\subsection{Experiment 4: Appropriate Acquisition Times and Temporal Resolution}

One of the challenges in using multi temporal data is to provide early information about cultivation area and crop types for decision makers with a few images. Hence, at first only different sequential times of path 162 were selected to assess which acquisition times were suitable for early classification. Then classification was conducted on case 1 using different combinations of acquisition time sequences. The summary of using 8 combinations of acquisition times that yield acceptable results are given in Table 7.

Table 7. The (OA) and kappa of the RF classifier using combinations of acquisition times.

\begin{tabular}{|c|c|c|c|c|c|c|c|c|c|c|c|c|}
\hline $\begin{array}{l}\dot{0} \\
\mathbf{Z} \\
\dot{0} \\
\dot{0} \\
0\end{array}$ & 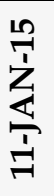 & 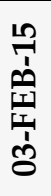 & 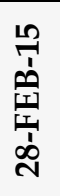 & 离 & $\sum_{\substack{1 \\
j}}^{\infty}$ & 足 & 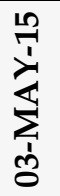 & 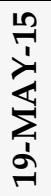 & 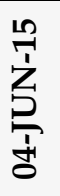 & 号 & $\overleftarrow{0}$ & 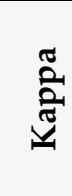 \\
\hline 1 & $\checkmark$ & - & $\checkmark$ & - & $\checkmark$ & $\checkmark$ & $\checkmark$ & $\checkmark$ & $\checkmark$ & $\checkmark$ & 93.2 & 85.3 \\
\hline 2 & $\checkmark$ & - & $\checkmark$ & - & $\checkmark$ & $\checkmark$ & $\checkmark$ & $\checkmark$ & $\checkmark$ & - & 93.1 & 85.2 \\
\hline 3 & $\checkmark$ & - & $\checkmark$ & - & $\checkmark$ & $\checkmark$ & $\checkmark$ & $\checkmark$ & - & - & 95.5 & 90.4 \\
\hline 4 & $\checkmark$ & - & $\checkmark$ & - & $\checkmark$ & $\checkmark$ & $\checkmark$ & - & - & - & 94.3 & 87.8 \\
\hline 5 & $\checkmark$ & - & $\checkmark$ & - & $\checkmark$ & $\checkmark$ & - & - & - & - & 91.2 & 81.2 \\
\hline 6 & $\checkmark$ & - & $\checkmark$ & - & $\checkmark$ & - & - & - & - & - & 86.8 & 71.5 \\
\hline 7 & $\checkmark$ & - & - & - & - & $\checkmark$ & - & - & - & - & 94.1 & 87.5 \\
\hline 8 & $\checkmark$ & - & - & - & - & $\checkmark$ & $\checkmark$ & - & - & - & 95.3 & 90.1 \\
\hline 9 & $\checkmark$ & $\checkmark$ & $\checkmark$ & $\checkmark$ & - & - & - & - & - & - & 87.4 & 72.8 \\
\hline
\end{tabular}

The superior result is obtained in Comb.3 when 6 images are used (Table 7). In contrast, an acceptable accuracy in the early season is obtained for decision makers when only the first three images of all images are used (i.e. Comb.6). Moreover, a suitable result is also achieved when only 2 images are used (i.e. Comb.7). The results may seem remarkable from the processing time standpoint due to using only 2 images.

To demonstrate the effect of increasing the temporal resolution of LDCM to one week, the images of path 163 (i.e. dates 03-FEB-15 and 07-MAR-15) are incorporated into the experiment. Therefore, the temporal resolution of LDCM on case study area (i.e. overlap area of two paths of Landsat 8) increased to one week. The experimental results demonstrated that, in this case study, the one-week temporal resolution of LDCM (i.e. Comb. 9), compared to two weeks temporal resolution of LDCM (i.e. Comb.6) achieved better results in terms of OA and kappa. This is due to the fact that only two images from path 163 captured in the first steps of growing season are included. Moreover, early classification (i.e. $07^{\text {th }}$ of March) with better result can be yield if the temporal resolution of Landsat increases to one week. This is promising so that more images with high temporal resolution like Sentinel2A and Sentinel2B can be employed. Although using a constellation of satellites with high spatial and temporal resolution increases the accuracy, certainly a balance between some factors like ground sampling size, processing time, accuracy and early season crop area estimation should be considered. 


\subsection{Experiment 5: The Effect of VIs and their Gradient}

This experiment aims at demonstrating the effect of VIs and their gradients on the classification accuracy improvement and their capabilities to decrease scheduled time for image acquisition. For this purpose, at first NDVI and STVI3 as examples of VIs are considered and temporal signatures of wheat and barley are computed and depicted in Figure 6 for training data. As shown in Fig. 6 wheat and barley have similar reflectance in the early stage of growing, making their discrimination difficult in early season. In contrast to wheat, barley yields faster and loses its chlorophyll almost 3 weeks earlier. Then temporal gradient of wheat and barley is computed and given in Figure 6. Although the temporal signatures of these crops in the first stages of growing is almost the same and have high spectral similarity, as shown in Fig. 6, the temporal gradient at the end of the season can provide appropriate features for better discrimination. In particular, the temporal gradient between images of T6 and T7 which acquired at 17 Apr. and 3 May respectively, could provide the first significant difference between these similar classes for discrimination. In other words, from 17 Apr. to 3 May the wheat stands on the late stage of its grows while barely lost chlorophyll more rapidly.

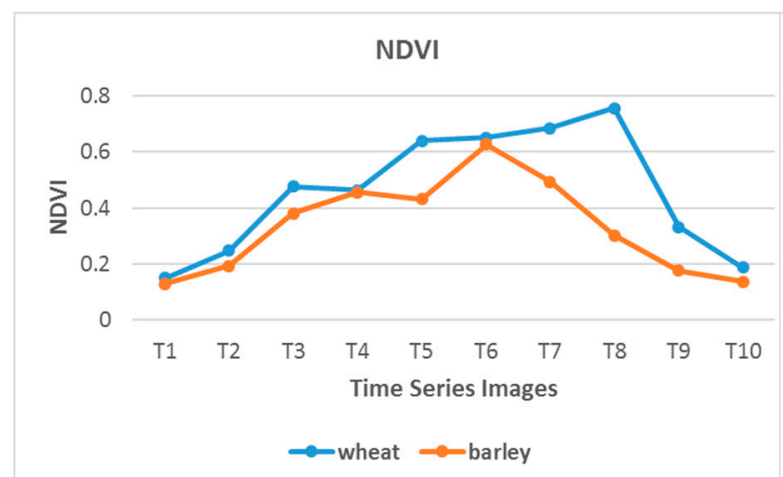

(a)

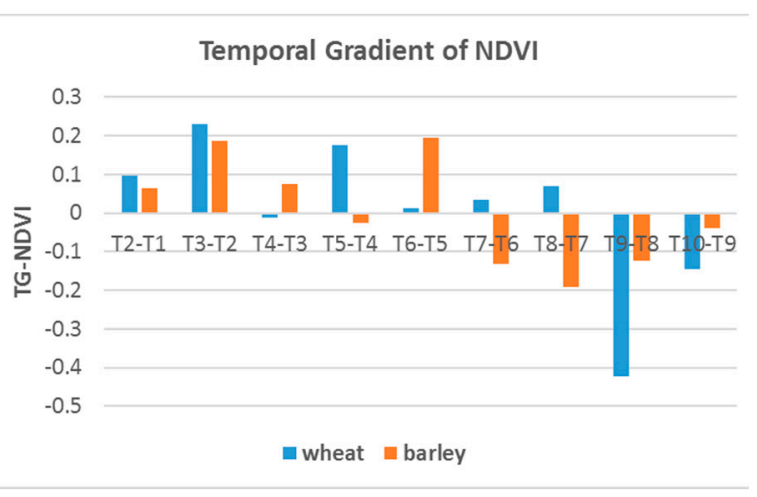

(b)

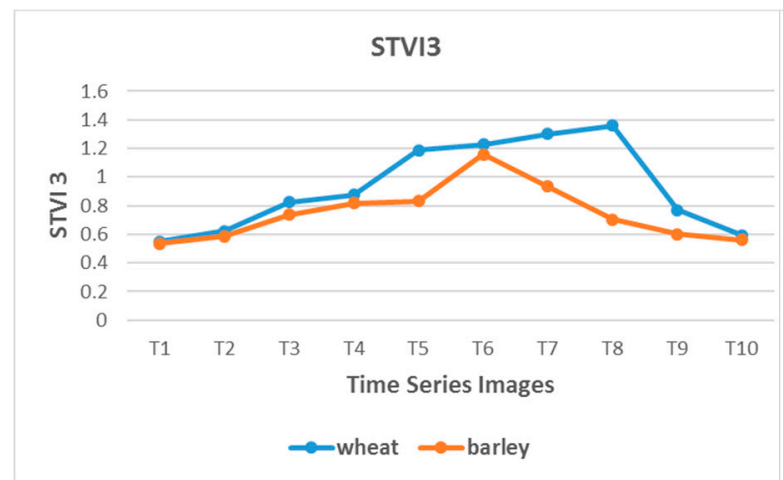

(c)

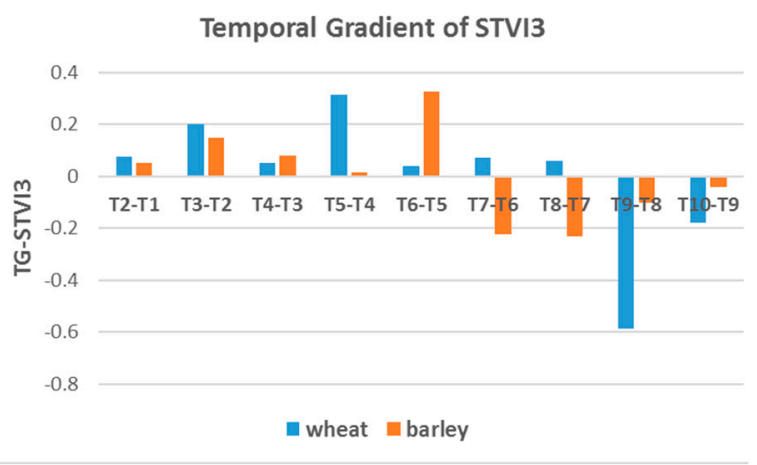

(d)

Figure 6. The NDVI and STVI3 indices and their temporal gradient for: (a) NDVI; (b) STVI3; (c) temporal gradient of NDVI; (d) temporal gradient of STVI3, during growing season.

After illustrating the VIs and their temporal gradients, the gradient of spectral bands and the aforementioned VIs (Table 2) along with their temporal gradients (i.e. Eqs. (10 \& 11)) are incorporated in the RF classification. Figure 6 and Figure 7 shows the results obtained for 28 combinations of acquisition times when only spectral bands are used. In addition to spectral bands, the effect of incorporating VIs and their spectral gradients obtained by Eq. (10) and Eq. (11) is given in Fig.7 in terms of $\mathrm{OA}$ and Kapa coefficient. As demonstrated in Fig. 7 by utilizing the temporal gradient of spectral bands and VIs along with their gradients the OA and Kappa are improved up to $5 \%$ and $9 \%$, respectively. 


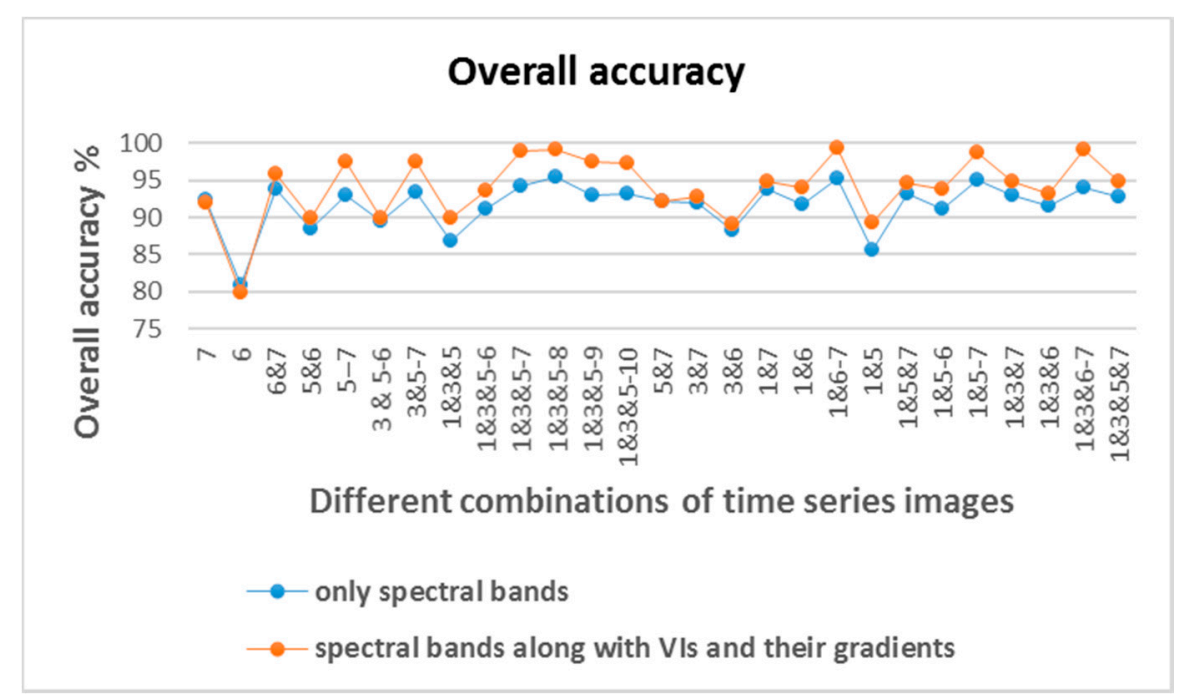

(a)

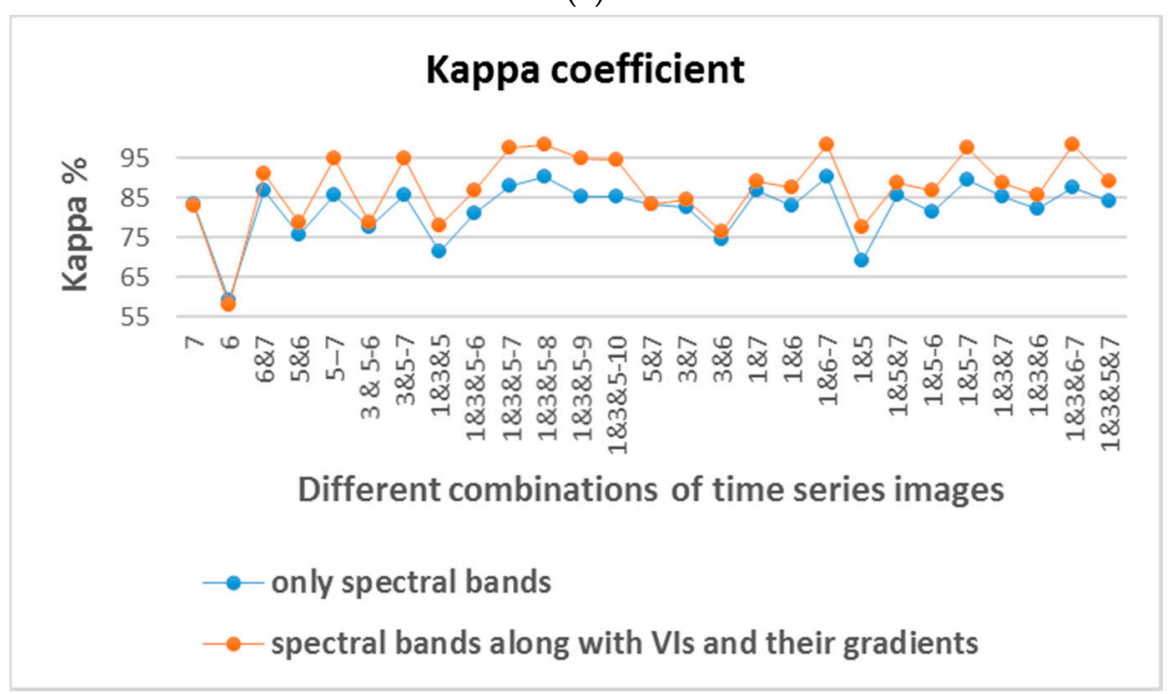

(b)

298 Figure 7. The classification results when only spectral bands are used versus spectral bands along with VIs and their gradients are used: (a) overall accuracy (OA). (b) Kapa coefficient

Table 8. The overall accuracy (OA), kappa and producer's accuracy (PA) of appropriate times with their Vis and gradient by the RF classifier.

\begin{tabular}{|c|c|c|c|c|c|c|c|c|c|c|c|c|c|c|c|c|c|}
\hline $\begin{array}{l}\dot{0} \\
\dot{z} \\
\dot{0} \\
\dot{0}\end{array}$ & $\frac{\stackrel{1}{T}}{\stackrel{i}{z}}$ & 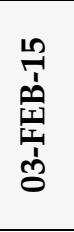 & 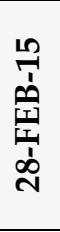 & 足 & 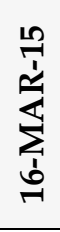 & 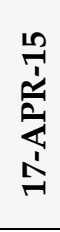 & 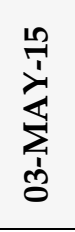 & 斈 & $\frac{\stackrel{n}{7}}{\stackrel{3}{z}}$ & 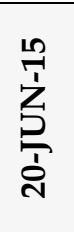 & $\stackrel{\infty}{>}$ & 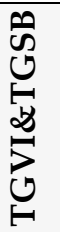 & $\overleftarrow{0}$ & $\frac{\tilde{a}}{\tilde{a}}$ & 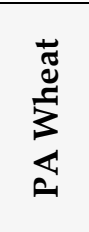 & 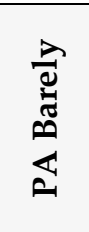 & $\frac{\grave{d}}{\square}$ \\
\hline 1 & $\checkmark$ & - & $\checkmark$ & - & $\checkmark$ & - & - & - & - & - & - & - & 86.8 & 71.5 & 82.4 & 9.8 & 95.9 \\
\hline 2 & $\checkmark$ & - & $\checkmark$ & - & $\checkmark$ & - & - & - & - & - & $\checkmark$ & - & 88.1 & 73.9 & 83.9 & 10.9 & 96.7 \\
\hline 3 & $\checkmark$ & - & $\checkmark$ & - & $\checkmark$ & - & - & - & - & - & $\checkmark$ & $\checkmark$ & 89.9 & 78.1 & 86.8 & 13.0 & 98.5 \\
\hline 4 & $\checkmark$ & - & - & - & - & $\checkmark$ & $\checkmark$ & - & - & - & - & - & 95.3 & 90.1 & 97.7 & 45.5 & 99.0 \\
\hline 5 & $\checkmark$ & - & - & - & - & $\checkmark$ & $\checkmark$ & - & - & - & $\checkmark$ & - & 96.4 & 92.3 & 98.2 & 49.3 & 100 \\
\hline 6 & $\checkmark$ & - & - & - & - & $\checkmark$ & $\checkmark$ & - & - & - & $\checkmark$ & $\checkmark$ & 99.3 & 98.4 & 99.4 & 92.5 & 99.9 \\
\hline 7 & $\checkmark$ & $\checkmark$ & $\checkmark$ & $\checkmark$ & - & - & - & - & - & - & $\checkmark$ & $\checkmark$ & 90.5 & 79.8 & 90.2 & 13.8 & 98.2 \\
\hline
\end{tabular}


302

303

304

305

306

307

308

309

310

311

312

313

314

315

316

317

318

319

320

321

322

323

324

325

326

327

328

329

330

331

332

333

334

335

From 28 combinations, 3 combinations which incorporate minimum acceptable number of images such that discriminate classes of case 1 in early season are selected for comparison. To demonstrate the impact of VIs and their gradients the OA, Kappa and product accuracies (PA) of classes are given in Table 8. As shown, when VIs added to comb. 1 (i.e. early season with 2 weeks temporal resolution) as new features the OA and Kappa improves $1.3 \%$. While this improvement is $1.8 \%$ when utilizing TGVI and TGSB. Therefore, the use of VIs, TGVI and TGBS as new features the OA and Kappa improve $3.1 \%$ and $6.6 \%$, respectively. Moreover, when VIs, TGVI and TGBS features added in Comb.4 not only improve the OA and Kappa but also the TGVI and TGBS features particularly discriminate barley from wheat and improves the PA of barley $43.2 \%$. In contrast to Comb.4, in Comb.3 the TGVI and TGBS do not improve PA of barley significantly. This is due to the similarity between wheat and barley in the early stage of growing. Therefore, the results demonstrated that the appropriate model is Comb.6 which utilizes only 1 image from the first stage of growing season and two images from seasonal change point of wheat and barley. It is worthy to note that compared to Comb.3 the scheduled time decreases to 07-MAR-15 in Comb.7 and yield better results in terms of $\mathrm{OA}$ and kappa when temporal resolution increase to one week (i.e. two images of path 163 are used).

\subsection{Experiment 6: The Effect of the Number of Classes}

In practice, the classification of all crop types is time and cost consuming. Also when different scenarios for image classification arise in terms of the number of classes, different classification accuracies can be obtained. Moreover, discriminating two similar classes like wheat and barley demands images from a seasonal change point in the temporal signature of those crops. On the other hand, decision makers are usually demand to get information about the cultivated area in early season even for only one or two crop types. Therefore, the number of desired classes to be determined effect on early season detection by the RF classifier. In this experiment, the RF classification is performed on classification case 2 of Table 5 using the appropriate models obtained through experiment 5 (i.e. Comb.3, Comb.6 and Comb.7 of Table 8). The comparative results are given in Table 9. The achieved results demonstrated that, compared to the classification case 1 , the classification accuracy increased up to $7 \%$ in case 2 in terms of OA (i.e. when two classes of wheat and barley are merged into one class). As a result, when decision makers demand to distinguish cultivate area of both wheat and barley, the Comb.7 is an appropriate model which utilizes 4 images from the first stage of growing season with one weak temporal resolution. In this regard, the crop area (Wheat along with Barely) can be estimated with appropriate results almost two months earlier (Table 9).

Table 9. The overall accuracy (OA), kappa and producers' accuracy (PA) when wheat and barley are merged to one class by the RF classifier.

\begin{tabular}{|c|c|c|c|c|c|c|c|c|c|c|c|c|c|c|c|c|}
\hline 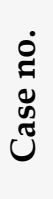 & 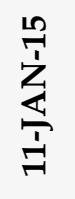 & $\begin{array}{l}\frac{10}{7} \\
\frac{0}{10} \\
\frac{11}{8} \\
8\end{array}$ & 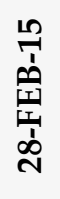 & 离 & $\sum_{\substack{1 \\
\vdots}}^{n}$ & $\begin{array}{l}\frac{10}{7} \\
\frac{\pi}{4} \\
\frac{1}{4} \\
\frac{1}{2}\end{array}$ & $\sum_{0}^{\infty}$ & 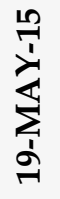 & 号 & 号 & $\stackrel{n}{>}$ & 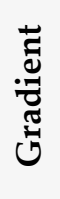 & $\overleftarrow{0}$ & $\frac{\tilde{a}}{\tilde{a}}$ & 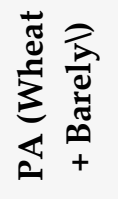 & $\begin{array}{l}\grave{\Xi} \\
\stackrel{\Xi}{0} \\
\overleftarrow{a}\end{array}$ \\
\hline 1 & $\checkmark$ & - & $\checkmark$ & - & $\checkmark$ & - & - & - & - & - & $\checkmark$ & $\checkmark$ & 95.9 & 90.7 & 92.1 & 97.8 \\
\hline 2 & $\checkmark$ & - & - & - & - & $\checkmark$ & $\checkmark$ & - & - & - & $\checkmark$ & $\checkmark$ & 99.6 & 99.0 & 99.0 & 99.8 \\
\hline 3 & $\checkmark$ & $\checkmark$ & $\checkmark$ & $\checkmark$ & - & - & - & - & 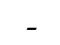 & - & $\checkmark$ & $\checkmark$ & 97.1 & 93.5 & 94.9 & 98.2 \\
\hline
\end{tabular}

\section{Conclusions}

In this study, multi-temporal images were used to discriminate wheat, barley and other classes by the RF classifier with the aims of using a few images acquired in the early stages of the growing season rather than using those of the entire growing season. In the study area, 10 images from the overlapping area between two over paths of 162 and 163 captured during 6 months were examined. 
The experimental results demonstrated that $30 \%$ of the data and 50 trees were suitable as the optimum training sample size and forest size, respectively in the study area. The miss values due to the cloudy pixels in multi-temporal images were retrieved by the average of the k-nearest cloudless pixels. The achieved results demonstrated that the OA decreased by $1.6 \%$ due to the retrieval miss values. Therefore, it is concluded that when miss values in the cloudy pixels are retrieved and classified by the RF classifier, the certainty of obtaining labels for these pixels is less than the certainty of labels of cloudless pixels. To determine appropriate acquisition times for identifying aforementioned classes, different combinations of acquisition times were generated and evaluated. The experiments demonstrated that the superior result was obtained when 6 images were used while an acceptable accuracy in early season was gained when only the first three images of all images were utilized. To demonstrate the effect of increasing the temporal resolution, the images of path 163 were incorporated into the experiment. The experimental results showed that if temporal resolution of Landsat 8 increased to one week the classification task can be conducted earlier with almost better results in terms of OA and kappa.

Furthermore, VIs along with TGVI and TGSB were generated as informative features to discriminate wheat and barley. In this regard, the impact of incorporating these generated features was assessed with the aim of decreasing period of time series and improving the accuracy. The results demonstrated that utilizing these new features improve the OA and Kappa 3.1\% and 6.6\%, respectively. Furthermore, the obtained result showed that TGVI and TGSB play the main role to discriminate remarkably wheat from barley if only one image from seasonal changes of crops is available. The experiments also demonstrated that if the early season detection of both wheat and barley as one class rather more important than separate them, the crop area can be estimated two months earlier with 97.1 and 93.5 in terms of OA and kappa, respectively.

The results obtained by this study are promising such that images with high temporal resolution like Sentinel2A and Sentinel2B can be used to achieve accurate discrimination in the early stage of the growing season. Although using a constellation of satellites with high spatial and temporal resolutions increases the accuracy, a balance between some factors like ground sampling size, processing time, accuracy and early season crop area estimation should be certainly considered.

Author Contributions: All the authors listed contributed equally to the work presented in this paper.

Conflicts of Interest: The authors declare no conflict of interest.

\section{References}

1. Godfray, H.C.J.; Beddington, J.R.; Crute, I.R.; Haddad, L.; Lawrence, D.; Muir, J.F.; Pretty, J.; Robinson, S.; Thomas, S.M.; Toulmin, C. Food security: The challenge of feeding 9 billion people. science 2010, 327, 812-818.

2. Padilla, F.; Maas, S.; González-Dugo, M.; Mansilla, F.; Rajan, N.; Gavilán, P.; Domínguez, J. Monitoring regional wheat yield in southern spain using the grami model and satellite imagery. Field Crops Research 2012, 130, 145-154.

3. Allen, R.; Hanuschak, G.; Craig, M. History of remote sensing for crop acreage in usda's national agricultural statistics service. 2002.

4. Pan, Y.; Li, L.; Zhang, J.; Liang, S.; Zhu, X.; Sulla-Menashe, D. Winter wheat area estimation from modis-evi time series data using the crop proportion phenology index. Remote Sensing of Environment 2012, 119, 232-242.

5. Ghamisi, P.; Plaza, J.; Chen, Y.; Li, J.; Plaza, A. Advanced supervised spectral classifiers for hyperspectral images: A review. IEEE Geoscience and Remote Sensing Magazine (GRSM) 2017. 
386 6. Vieira, C.; Mather, P.; McCULLAGH, M. The spectral-temporal response surface

387

388

389

390

391

392

393

394

395

396

397

398

399

400

401

402

403

404

405

406

407

408

409

410

411

412

413

414

415

416

417

418

419

420

421

422

423

424

425

426

427

428 and its use in the multi-sensor, multi-temporal classification of agricultural crops. International Archives of Photogrammetry and Remote Sensing 2000, 33, 582-589.

7. Wardlow, B.D.; Egbert, S.L.; Kastens, J.H. Analysis of time-series modis $250 \mathrm{~m}$ vegetation index data for crop classification in the us central great plains. Remote Sensing of Environment 2007, 108, 290-310.

8. Cammarano, D.; Fitzgerald, G.J.; Casa, R.; Basso, B. Assessing the robustness of vegetation indices to estimate wheat $\mathrm{n}$ in mediterranean environments. Remote Sensing 2014, 6, 2827-2844.

9. de Colstoun, E.C.B.; Story, M.H.; Thompson, C.; Commisso, K.; Smith, T.G.; Irons, J.R. National park vegetation mapping using multitemporal landsat 7 data and a decision tree classifier. Remote Sensing of Environment 2003, 85, 316-327.

10. Langley, S.K.; Cheshire, H.M.; Humes, K.S. A comparison of single date and multitemporal satellite image classifications in a semi-arid grassland. Journal of Arid Environments 2001, 49, 401-411.

11. Carrão, H.; Gonçalves, P.; Caetano, M. Contribution of multispectral and multitemporal information from modis images to land cover classification. Remote Sensing of Environment 2008, 112, 986-997.

12. Chen, J.; Lu, M.; Chen, X.; Chen, J.; Chen, L. A spectral gradient difference based approach for land cover change detection. ISPRS journal of photogrammetry and remote sensing 2013, 85, 1-12.

13. Zheng, B.; Myint, S.W.; Thenkabail, P.S.; Aggarwal, R.M. A support vector machine to identify irrigated crop types using time-series landsat ndvi data. International Journal of Applied Earth Observation and Geoinformation 2015, 34, 103-112.

14. Bellón, B.; Bégué, A.; Lo Seen, D.; de Almeida, C.A.; Simões, M. A remote sensing approach for regional-scale mapping of agricultural land-use systems based on ndvi time series. Remote Sensing 2017, 9, 600.

15. Lobell, D.B.; Asner, G.P. Cropland distributions from temporal unmixing of modis data. Remote Sensing of Environment 2004, 93, 412-422.

16. Nitze, I.; Schulthess, U.; Asche, H. Comparison of machine learning algorithms random forest, artificial neural network and support vector machine to maximum likelihood for supervised crop type classification. Proc. of the 4th GEOBIA 2012, 79.

17. Lebourgeois, V.; Dupuy, S.; Vintrou, É.; Ameline, M.; Butler, S.; Bégué, A. A combined random forest and obia classification scheme for mapping smallholder agriculture at different nomenclature levels using multisource data (simulated sentinel-2 time series, vhrs and dem). Remote Sensing 2017, 9, 259.

18. Chen, J.; Jönsson, P.; Tamura, M.; Gu, Z.; Matsushita, B.; Eklundh, L. A simple method for reconstructing a high-quality ndvi time-series data set based on the savitzky-golay filter. Remote sensing of Environment 2004, 91, 332-344.

19. Potgieter, A.; Apan, A.; Hammer, G.; Dunn, P. Early-season crop area estimates for winter crops in ne australia using modis satellite imagery. ISPRS Journal of Photogrammetry and Remote Sensing 2010, 65, 380-387. 
20. Du, P.; Xia, J.; Zhang, W.; Tan, K.; Liu, Y.; Liu, S. Multiple classifier system for remote sensing image classification: A review. Sensors 2012, 12, 4764-4792.

21. Millard, K.; Richardson, M. On the importance of training data sample selection in random forest image classification: A case study in peatland ecosystem mapping. Remote sensing 2015, 7, 8489-8515.

22. Fletcher, R.S. Using vegetation indices as input into random forest for soybean and weed classification. American Journal of Plant Sciences 2016, 7.

23. Eisavi, V.; Homayouni, S.; Yazdi, A.M.; Alimohammadi, A. Land cover mapping based on random forest classification of multitemporal spectral and thermal images. Environmental monitoring and assessment 2015, 187, 1-14.

24. Pelletier, C.; Valero, S.; Inglada, J.; Champion, N.; Dedieu, G. Assessing the robustness of random forests to map land cover with high resolution satellite image time series over large areas. Remote Sensing of Environment 2016, 187, 156-168.

25. Nitze, I.; Barrett, B.; Cawkwell, F. Temporal optimisation of image acquisition for land cover classification with random forest and modis time-series. International Journal of Applied Earth Observation and Geoinformation 2015, 34, 136-146.

26. Hao, P.; Zhan, Y.; Wang, L.; Niu, Z.; Shakir, M. Feature selection of time series modis data for early crop classification using random forest: A case study in kansas, USA. Remote Sensing 2015, 7, 5347-5369.

27. Inglada, J.; Arias, M.; Tardy, B.; Hagolle, O.; Valero, S.; Morin, D.; Dedieu, G.; Sepulcre, G.; Bontemps, S.; Defourny, P. Assessment of an operational system for crop type map production using high temporal and spatial resolution satellite optical imagery. Remote Sensing 2015, 7, 12356-12379.

28. Liu, J.; Feng, Q.; Gong, J.; Zhou, J.; Liang, J.; Li, Y. Winter wheat mapping using a random forest classifier combined with multi-temporal and multi-sensor data. International Journal of Digital Earth 2017, 1-20.

29. Rouse JW, H.R., Schell JA, Deering DW Monitoring vegetation, systems in the great plains with erts. In Proceeding of Third Earth Resources,Technology Satellite Symposium 1, Greenbelt, USA 1974.

30. Jordan, C.F. Derivation of leaf-area index from quality of light on the forest floor. Ecology 1969, 50, 663-666.

31. Ridao, E.; Conde, J.R.; M'nguez, M.I. Estimating fapar from nine vegetation indices for irrigated and nonirrigated faba bean and semileafless pea canopies. Remote Sensing of Environment 1998, 66, 87-100.

32. Pearson, R.L.; Miller, L.D. In Remote mapping of standing crop biomass for estimation of the productivity of the shortgrass prairie, Remote Sensing of Environment, VIII, 1972; p 1355.

33. Thenkabail, P.S.; Ward, A.D.; Lyon, J.G.; Merry, C.J. Thematic mapper vegetation indices for determining soybean and corn growth parameters. Photogrammetric Engineering and Remote Sensing 1994, 60, 437-442.

34. Jafari, R.; Lewis, M.; Ostendorf, B. Evaluation of vegetation indices for assessing vegetation cover in southern arid lands in south australia. The Rangeland Journal 2007, 29, 39-49. 
472 35. Liu, H.Q.; Huete, A. A feedback based modification of the ndvi to minimize canopy

473

474

475

476

477

478

479

480

481

482

483

484

485

486

487

488

489

490

491

492

493 background and atmospheric noise. IEEE Transactions on Geoscience and Remote Sensing 1995, 33, 457-465.

36. Qi, J.; Chehbouni, A.; Huete, A.; Kerr, Y.; Sorooshian, S. A modified soil adjusted vegetation index. Remote sensing of environment 1994, 48, 119-126.

37. Nemani, R.; Pierce, L.; Running, S.; Band, L. Forest ecosystem processes at the watershed scale: Sensitivity to remotely-sensed leaf area index estimates. International journal of remote sensing 1993, 14, 2519-2534.

38. Huete, A.R. A soil-adjusted vegetation index (savi). Remote sensing of environment 1988, 25, 295-309.

39. Freund, Y.; Schapire, R.E. In Experiments with a new boosting algorithm, Icml, 1996; pp 148-156.

40. Leistner, C.; Saffari, A.; Santner, J.; Bischof, H. In Semi-supervised random forests, 2009 IEEE 12th International Conference on Computer Vision, 2009; IEEE: pp 506513.

41. Breiman, L. Out-of-bag estimation; Citeseer: 1996.

42. Breiman, L. Random forests. Machine learning 2001, 45, 5-32.

43. Congalton, R.G.; Green, K. Assessing the accuracy of remotely sensed data: Principles and practices. CRC press: 2008.

44. Wang, L.a.; Zhou, X.; Zhu, X.; Dong, Z.; Guo, W. Estimation of biomass in wheat using random forest regression algorithm and remote sensing data. The Crop Journal 2016, 4, 212-219. 